\title{
Efficacy, safety and administration timing of trastuzumab in human epidermal growth factor receptor 2 positive breast cancer patients: A meta-analysis
}

\author{
YUAN-YUAN CHEN, LIN-WEI WANG, FANG-FANG CHEN, BI-BO WU and BIN XIONG \\ Department of Oncology, Zhongnan Hospital of Wuhan University, \\ Hubei Key Laboratory of Tumor Biological Behaviors and Hubei Cancer Clinical Study Center, \\ Wuhan, Hubei 430071, P.R. China
}

Received December 5, 2014; Accepted January 11, 2016

DOI: 10.3892/etm.2016.3095

\begin{abstract}
Trastuzumab has been demonstrated to be an effective treatment in patients with human epidermal growth factor receptor-2 (HER-2) positive breast cancer (BC); however, inconsistent results with regards to the long-term survival benefits, safety and optimal administration timing of trastuzumab exist. The present meta-analysis investigated these inconsistencies in patients with HER-2 positive BC that received adjuvant or neoadjuvant trastuzumab. Computerized and manual searches were used to identify eligible randomized control trials (RCTs) to include in the analysis. Based on a fixed or random effects model, hazard and risk ratios were calculated and used to assess the survival advantages and risks of trastuzumab. A total of 14,546 patients from 13 RCTs were included in the analysis; 9 RCTs used an adjuvant setting and 4 RCTs used a neoadjuvant setting. Analysis of RCTs with an adjuvant setting demonstrated that treatment with trastuzumab and chemotherapy in patients with HER-2 positive $\mathrm{BC}$, in comparison with patients receiving chemotherapy alone, improved disease-free survival, overall
\end{abstract}

Correspondence to: Professor Bin Xiong, Department of Oncology, Zhongnan Hospital of Wuhan University, Hubei Key Laboratory of Tumor Biological Behaviors and Hubei Cancer Clinical Study Center, 169 Donghu Road, Wuchang, Wuhan, Hubei 430071, P.R. China

E-mail: binxiong19@163.com

Abbreviations: BC, breast cancer; HER-2, human epidermal growth factor receptor-2; RCTs, randomized control trials; AEs, adverse events; LVEF, left ventricular ejection fraction; CHF, congestive heart failure; DFS, disease-free survival; OS, overall survival; $\mathrm{CR}$, complete response; $\mathrm{PR}$, partial response; $\mathrm{SD}$, stable disease; PD, progressive disease; OR, overall response; HR, hazard ratio; $\mathrm{RR}$, risk ratio; $\mathrm{CI}$, confidence interval; T-group, trastuzumab plus chemotherapy group; C-group, chemotherapy group

Key words: breast cancer, meta-analysis, human epidermal growth factor receptor-2, trastuzumab survival and overall response. However, a higher incidence of neutropenia $(\mathrm{P}<0.0001)$, leukopenia $(\mathrm{P}<0.0001)$, diarrhea $(\mathrm{P}=0.002)$, skin/nail change $(\mathrm{P}=0.02)$, left ventricular ejection fraction reduction $(\mathrm{P}=0.007)$ and congestive heart failure $(\mathrm{P}<0.00001)$ was observed. Notably, the incidence of mortality and cardiac toxicity following concurrent and weekly use of trastuzumab was significantly lower compared to treatment with trastuzumab sequentially and every 3 weeks, respectively. Additionally, trastuzumab improved the pathologic complete response with no additional toxicity in the neoadjuvant setting. The present meta-analysis summarizes that trastuzumab is efficacious in patients with HER-2 positive BC in adjuvant and neoadjuvant settings. Thus, concurrent and weekly administration of trastuzumab is preferable to treatment with trastuzumab sequentially and every 3 weeks. These findings should be considered when using trastuzumab in future clinical practice.

\section{Introduction}

Breast cancer (BC) is the most common form of malignant tumor and is a leading cause of mortality in women worldwide (1). However, the recurrence and mortality of patients with BC has markedly decreased as a result of improvement in comprehensive treatments, in particular the use of adjuvant therapies including cytotoxic drugs, endocrine therapy and more recently trastuzumab, a monoclonal antibody against the human epidermal growth factor receptor-2 (HER-2) gene (2). The survival benefit gained from chemotherapy in BC patients has been observed to be equal regardless of whether chemotherapy was administered pre- or postoperatively (3). HER-2, also known as HER2/neu or c-erb-B2, encodes a 185-kD transmembrane glycoprotein receptor (4). Overexpression of the HER-2 gene has been detected in $25-30 \%$ patients with BC and is related to the aneuploidy growth of $\mathrm{S}$ phase, high nuclear grade, positive axillary node extension and low expression of hormonal receptors (5-8). In addition, the overexpression of HER-2 may lead to the activation of HER-2 signal pathways and uncontrolled cell differentiation (9). Therefore, HER-2-positive tumors have more aggressive biological behavior, and reoccur and undergo metastasis more readily compared with HER-2-negative tumors $(4,7,8)$. 
As a targeted drug, trastuzumab is recommended by the Food and Drug Administration to treat patients with HER-2 positive BC (10). A number of randomized control trials (RCTs) investigating the safety and efficacy of trastuzumab have demonstrated that a combination of trastuzumab and traditional drugs for chemotherapy results in a higher survival and response rate compared with chemotherapy alone in an adjuvant setting (11-13). In addition, trastuzumab may provide additional clinical benefits by increasing the rates of pathologic responses and breast-conserving therapy in a neoadjuvant setting $(14,15)$.

Despite this, a number of limitations still exist regarding the administrated of trastuzumab; for example, it is not fully understood how to optimize the treatment of combined trastuzumab and chemotherapy. Yin et al (16) reported that concurrent administration of trastuzumab resulted in a lower risk of mortality compared with sequential administration. By contrast, Azim et al (17) reported that no significant difference was observed in the overall survival (OS) rate between the two treatment options. Currently, it is unknown how to obtain the highest survival rates by changing the timings of trastuzumab administration.

It has been demonstrated that the successful administration of trastuzumab is associated with severe adverse effects (AEs) including cardiac toxicities and brain metastases (18-20). However, the presence of serious AEs in a number of organs has not been studied extensively. In addition, it is difficult to evaluate the efficacy and safety of trastuzumab due to the limited number of studies and quantity of data provided in previous meta-analyses $(11,14,16,19)$. Furthermore, odds ratios and relative risk (RR) are less appropriate and unreliable for analyzing time-to-event outcomes, as survivors or recovered individuals in the treatment group are simply compared with those in a control group at a single point in time (21). Therefore, based on the publication of several high quality RCTs in recent years, an updated meta-analysis was performed in the present study in order to evaluate the prognostic effects and the magnitude of AEs caused by trastuzumab in adjuvant and neoadjuvant settings.

\section{Materials and methods}

Search strategy and eligibility criteria. PubMed (http://www. ncbi.nlm.nih.gov/pubmed), Embase (http://www.embase. com/info/helpfiles/) and Science Direct (http:/www.elsevier. com/online-tools/sciencedirect) databases were used to identify eligible studies published between January 1995 and March 2014. Searched keywords included the following terms: 'Breast cancer', 'trastuzumab', 'herceptin', 'HER-2', 'adjuvant', 'neoadjuvant', 'chemotherapy' and 'random'. The reference lists of previous published meta-analyses were manually searched and reviews were collected without language restriction. Only RCTs that evaluated the efficacy or safety of chemotherapy with trastuzumab in patients with HER-2 positive BC were selected for inclusion in the present meta-analysis. Patients included in the current study required a good performance status (defined as a World Health Organization performance status of 0 or 1), adequate left ventricular ejection fraction (LVEF; as assessed by multiple-gated acquisition or echocardiography scan, and was required to be within the institutional normal range, and within the lower limit of normal), and normal bone marrow (a blood leukocyte count $>3.0 \times 10^{9}$ cells $/ 1$; neutrophil count $>1.5 \times 10^{9}$ cells $/ 1$; platelet count $>100 \times 10^{9}$ cells $/ 1$; hemoglobin $>10 \mathrm{~g} / \mathrm{dl}$ ), liver and renal function laboratory results. In the case of obtaining multiple reports of the same trial, the report with the longest follow-up period was selected for use. Patients who underwent chemotherapy were only compared with patients who received the same type of chemotherapy plus trastuzumab. Trials testing the administration of trastuzumab in a neoadjuvant setting were also included in this meta-analysis. The surgical modality, chemotherapy regimens, radiotherapy and endocrine therapy were not considered as eligibility criteria in selecting RCTs. RCTs which evaluated biological or targeted agents other than trastuzumab were excluded from the present study. The Preferred Reporting Items for Systematic Reviews and Meta-Analyses (22) checklist was adhered to in the present meta-analysis (data not shown).

Data extraction. Two reviewers independently extracted data from each trial included in the present study. Any discrepancies were resolved by discussing the problem with another author. The group of patients undergoing treatment with trastuzumab and chemotherapy were defined as the T-group, and patients only undergoing chemotherapy were defined as the C-group. The following information was obtained from chosen studies: Publication year, first author, patient follow-up time, chemotherapy regimens, number of recruited patients and the outcome events. The primary endpoint was the number of disease-free survival (DFS) patients, which was defined by events from the random assignment to the first documented disease progression (local, regional, distant recurrence or morality). Secondary endpoints included OS, tumor response and AEs. OS was defined as events from random assignment to mortality. The effects of treatment were assessed by the clinical response of the patient in an adjuvant setting, which included the complete response (CR), partial response (PR), stable disease (SD) and progressive disease (PD). The overall response (OR) was defined as CR plus PR. The effects of treatment in a neoadjuvant setting were divided into pathological CR (pCR) and non-pCR. AEs were graded according to the National Cancer Institute Common Toxicity Criteria (NCI-CTC) version 2.0 (23). Serious AEs were defined as grade 3-4 and fatal AEs were also included in the present study.

Quality assessment. The quality of the included trials was evaluated by the Cochrane Collaboration's risk of bias tool (24). This tool includes adequate sequence generation, allocation concealment, blinding of participants and personnel, blinding of outcome assessment, incomplete outcome data, selective outcome reporting and other bias. Each item is recorded as having a low, high or unclear risk of bias, and then a summary assessment of each included trial is graded as A, minimization of bias in all four categories: Adequate randomization, few losses to follow up and intention-to-treat analysis, blinding of outcome assessors, high quality outcome assessment; B, each of the criteria in 'A' partially met and $\mathrm{C}$, one or more of the criteria in 'A' not met. Two reviewers checked the risk of bias concurrently. 
Statistical analysis. Review Manager (Version 5.2; Copenhagen, Denmark: The Nordic Cochrane Centre, The Cochrane Collaboration, 2012) was used to perform the statistical analysis. The hazard ratio (HR) and $95 \%$ confidence interval (CI) were used to assess the survival advantage in patients with HER-2 positive BC. If provided in the trial, the P-value and HR from the Cox regression model was recorded and used directly in the present meta-analysis. If it was not available, an HR approximation was calculated indirectly using the methods described by Tierney et al (21). The risk ratio (RR) was calculated to estimate the relative risk of response and AEs. Statistical heterogeneity among the included studies was evaluated using the $\chi^{2}$ test and quantified using $\mathrm{I}^{2}$ statistics that determined the use of the fixed-effects (Mantel-Haenszel method) or random-effects (DerSimonian and Laird method) model (25). The existence of homogeneity was considered unreasonable when $\mathrm{I}^{2}>50 \%$ and $\mathrm{P}<0.10$. The comparison of trastuzumab stratified analysis was obtained by adjusted indirect comparison using the Bucher's method (26) and indirect treatment comparison (ITC) software (version 1.0; Canadian Agency for Drugs and Technologies in Health, Ottawa, Canada) that estimated the relative effects of concurrent trastuzumab to sequential trastuzumab, weekly trastuzumab to every 3 weeks trastuzumab through C-group, as previously described $(27,28)$. Finally, potential publication bias was evaluated using funnel plots and further quantified by Begg and Egger's tests $(29,30)$ using STATA software (version 12.0; College Station, TX, USA). $\mathrm{P}<0.05$ was considered to indicate a statistically significant difference.

\section{Results}

Characteristics of included clinical trials. A total of 419 studies were obtained from databases and 10 studies were obtained from references. Based on above criteria, 13 RCTs were included in the analysis, including 9 RCTs with adjuvant settings (31-39) and 4 with neoadjuvant settings (40-43). One joint trial (32) which contained two similar studies [NCCTG N9831 (18) and NSABP B-31 (20)] was included in the meta-analysis. Longer follow-up and detailed AEs were reported by Buzdar et al $(43,44)$; therefore, the two studies were combined in the analysis. The screening process that was used is briefly described in Fig. 1.

A total of 14,546 patients were available for this meta-analysis (31-43), of which 14,056 patients received adjuvant therapy and 490 patients received neoadjuvant therapy. In the adjuvant setting, 8,418 patients were assigned to the T-group and 5,638 patients were assigned to the C-group. In the neoadjuvant setting, 246 patients were treated with combination therapy and 244 were treated with chemotherapy alone. The HER-2 status of patients was assessed by immunohistochemistry, and fluorescence in situ hybridization or chromogenic in situ hybridization. Among the trials included in the present meta-analysis, the following trastuzumab treatment plans were used: An intravenous initial dose of $4 \mathrm{mg} / \mathrm{kg}$, followed by $2 \mathrm{mg} / \mathrm{kg}$ once a week, was used in 6 trials $(32,36-39,43)$; an initial dose of $8 \mathrm{mg} / \mathrm{kg}$, then $6 \mathrm{mg} / \mathrm{kg}$ every 3 weeks, was used in 5 trials $(33,35,40-42)$; in 2 trials, trastuzumab was administered initially at $4 \mathrm{mg} / \mathrm{kg}$, followed by $2 \mathrm{mg} / \mathrm{kg}$ per week, and

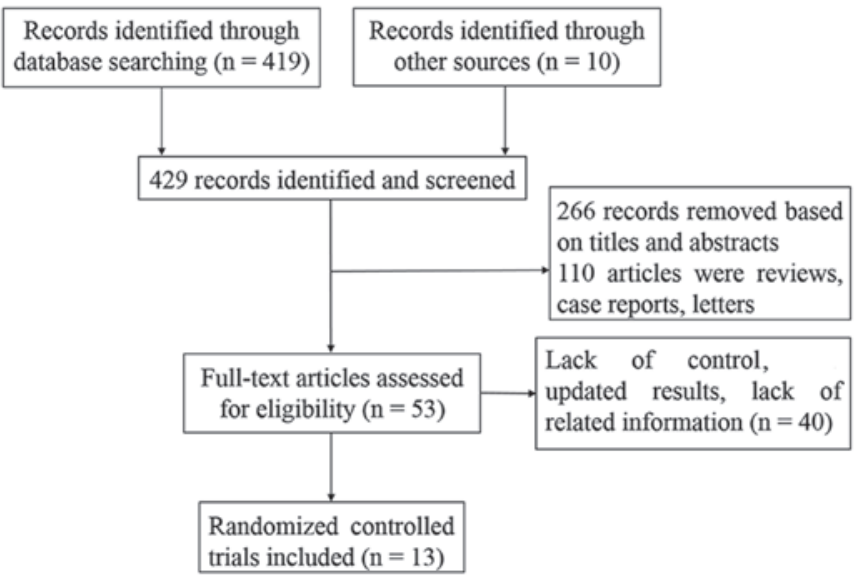

Figure 1. Flow chart of the screening process.

then $6 \mathrm{mg} / \mathrm{kg}$ every 3 weeks (31) or given at $6 \mathrm{mg} / \mathrm{kg}$ every 3 weeks (34). The median follow-up time ranged between 15.6 and 65.0 months. Baseline characteristics of the RCTs are summarized in Table I.

Methodological quality assessment. Each trial included a statement regarding randomization and a detailed description was included in 7 trials. One study was randomized using a code envelope (37), two by computer program $(40,41)$, and four via block method $(34,36,38,43)$. Two trials utilized the blind method $(38,42)$. All trials published as full text articles were judged to be grade B.

Efficacy and overall analysis of trastuzumab on DFS and OS in an adjuvant setting. In an adjuvant setting, 6 trials (31-36) with 10,503 patients reported disease recurrence. The random effects model $\left(\mathrm{I}^{2}=70 \%\right)$ indicated that the disease recurrence risk in C group $(26.1 \% ; 1,370 / 5,246)$ was higher compared that in the T group $[18.8 \% ; 987 / 5,257 ; \mathrm{HR}=0.66$; 95\% CI (0.58-0.75), $\mathrm{P}<0.00001$; Fig. 2A]. There were 8 studies $(30-35,37,38)$ with complete information on OS. In comparison with the C-group, there was a significant relative reduction in the risk of mortality in the T-group [21\%; $\mathrm{HR}=0.79 ; 95 \% \mathrm{CI}(0.68-0.92) ; \mathrm{P}=0.002 ; \mathrm{I}^{2}=62 \%$; Fig. $\left.2 \mathrm{~B}\right]$.

Subgroup analysis of trastuzumab on DFS and OS in an adjuvant setting. To further analyze the effects of different time schedules of trastuzumab administration on the survival rate, patients were divided into concurrent and sequential groups, then into patients treated weekly and every 3 weeks. With regards to DFS, the effect of each factor was consistent with the overall result (Fig. 3A). ITC demonstrated that there was no statistical difference between the concurrent and sequential groups $(\mathrm{RR}=0.779 ; \mathrm{P}=0.06059)$, but that the risk of recurrence following a weekly treatment plan was lower than the risk of patients on a 3 weekly treatment plan ( $R R=0.697 ; P=0.01128)$. However, the effect of trastuzumab on OS differed between the T-group and C-group; concurrent $(\mathrm{RR}=0.75 ; \mathrm{P}=0.003)$ and weekly $(\mathrm{RR}=0.78 ; \mathrm{P}=0.04)$ use of trastuzumab significantly lowered the risk of mortality in comparison with the $\mathrm{C}$-groups. The differences between the sequential and $\mathrm{C}$-group $(\mathrm{P}=0.18)$, and the 3 weekly treatment 


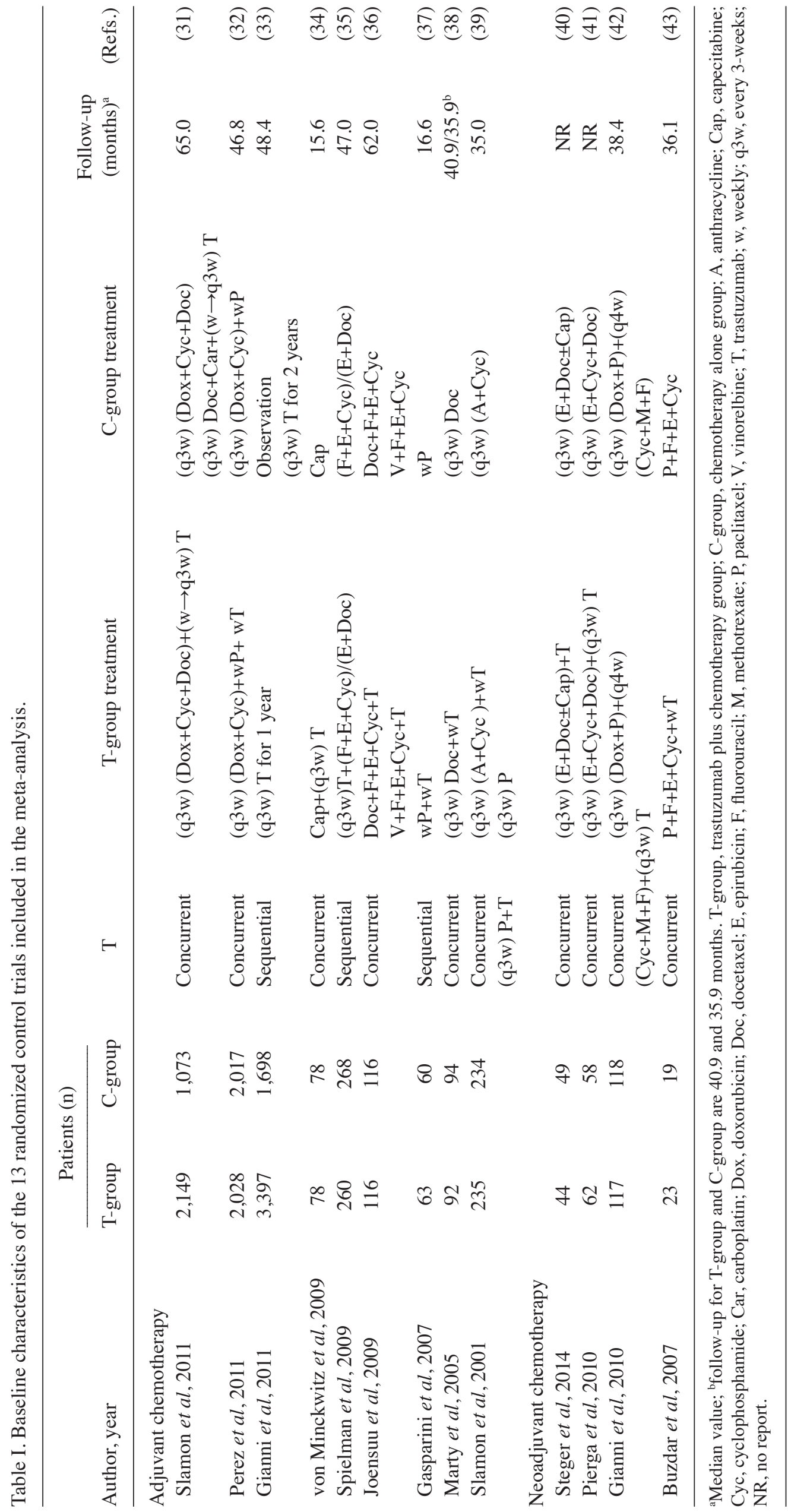




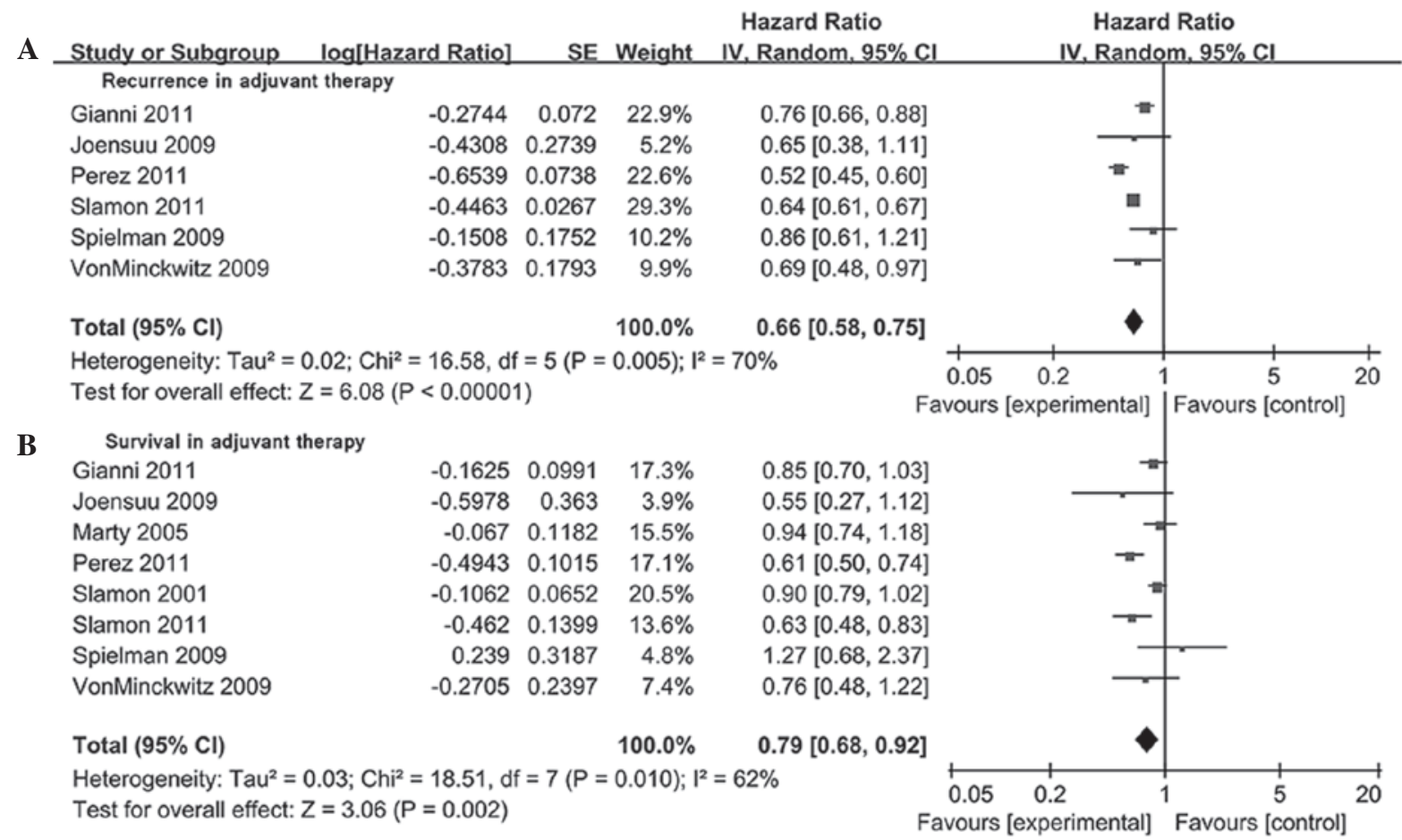

Figure 2. Forest-plot of the efficacy of trastuzumab in an adjuvant setting. Analysis of (A) recurrence and (B) survival between trastuzumab and the control group.
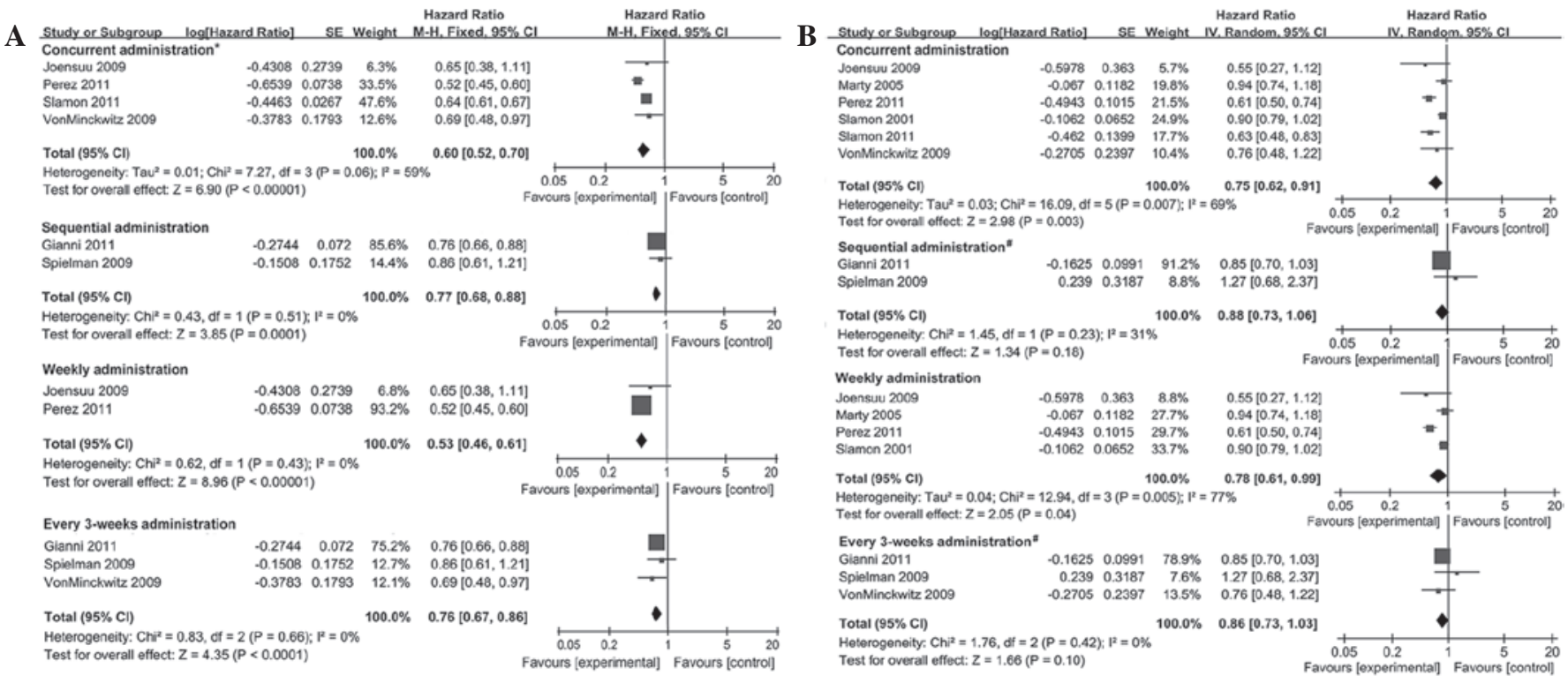

Figure 3. Forest plots of (A) disease free survival (B) overall survival in adjuvant chemotherapy setting by the timing of trastuzumab initiation. "Used random effect models; " used fixed effects model.

and $\mathrm{C}$-group $(\mathrm{P}=0.1)$ were not significantly different with regards to OS (Fig. 3B).

Overall analysis of trastuzumab on DFS and OS in a neoadjuvant setting. Only 2 RCTs contained survival analysis data in a neoadjuvant setting. The NOAH trial (42) demonstrated that the addition of trastuzumab could reduce the risk of relapse and mortality in comparison with the C-group. Buzdar et al observed that the DFS at 1 and 3 years was $100 \%$ in the
T-group ( $\mathrm{P}=0.041)$ (43). The data required to evaluate DFS and $\mathrm{OS}$ in neoadjuvant treatment groups was not available.

Response rates in adjuvant and neoadjuvant settings. Tumor response data was available in 4 adjuvant and 4 neoadjuvant trials. Among these, 3 trials (37-39) adopted the World Health Organization criteria (45) for the response measurement, 2 trials $(34,42)$ used the response evaluation criteria in solid tumors and 1 (41) adopted chevalier criteria. The criteria in a 


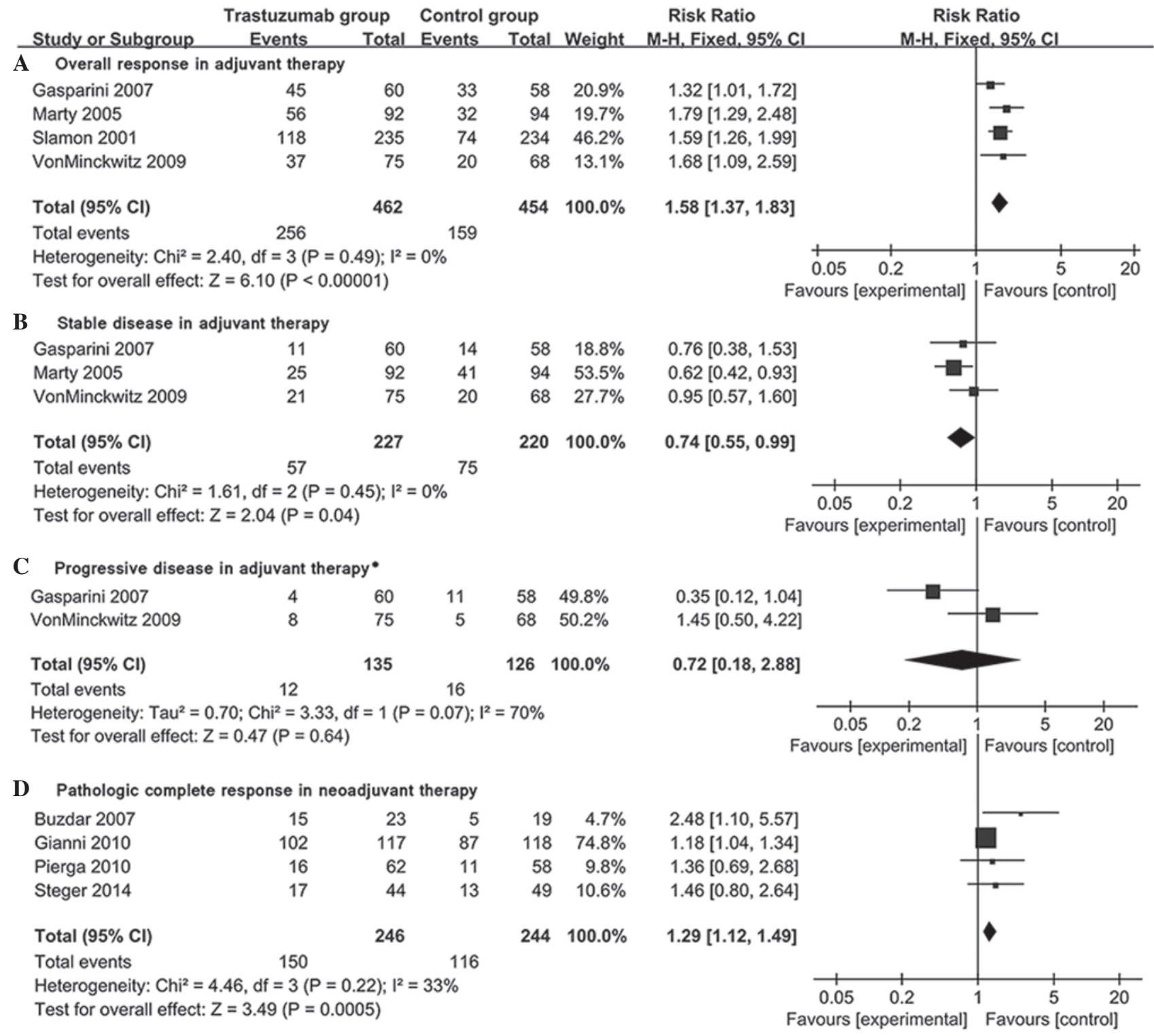

Figure 4. Forest-plots of the tumor response rate of (A) overall response (B) stable disease (C) progressive disease in an adjuvant setting and (D) pathologic complete response in a neoadjuvant setting. "Used random effect models.

further 2 trials were unclear $(40,43)$. These studies (34,37-43) were incorporated in the response analysis for relatively high concordance among these criteria (46). The OR, SD and PD was analyzed in 4, 3 and 2 adjuvant setting trials, respectively. The rate of OR was significantly higher in the T-group in comparison with the C-group [RR=1.58; 95\% CI (1.37-1.83); $\mathrm{P}<0.00001$; Fig. 4A). However, the rate of SD in the T-group was significantly lower than that in the $\mathrm{C}$-group $[\mathrm{RR}=0.74$, 95\% CI (0.55-0.99), P=0.04; Fig. 4B]. There was no statistical difference between the rate of PD between the two groups [RR=0.72; 95\% CI (0.18-2.88); P=0.64; Fig. 4C]. The estimated pCR in 4 neoadjuvant trials demonstrated that the $\mathrm{pCR}$ in the T-group $(61 \%$; 150/246) was significantly higher compared with the C-group [48\%; 116/244; RR=1.29; 95\% CI (1.12-1.49); $\mathrm{P}=0.0005$; Fig. 4D].

Safety. According to NCI-CTC 2.0, serious AEs reported in $\geq 2$ trials or with an incidence of $>5 \%$ were classified into different systems for analysis. Based on these criteria, serious
AEs in hematological and lymphatic systems, the digestive tract, the circulatory system, respiratory tract, nervous system, musculoskeletal system and others were studied in the present meta-analysis.

Serious AEs in an adjuvant setting. In comparison with the $\mathrm{C}$-group, patients in the T-group were more likely to suffer from neutropenia (61.9 vs. $54.2 \%, \mathrm{P}<0.0001)$, leukopenia (57.1 vs. $48.8 \%, \mathrm{P}<0.0001)$, diarrhea ( 2.9 vs. $1.6 \%, \mathrm{P}=0.002$ ), a decrease in $\operatorname{LVEF}$ (8.6 vs. $4.4 \%, \mathrm{P}=0.007$ ), congestive heart failure $(\mathrm{CHF})$ ( 2.4 vs. $0.4 \%, \mathrm{P}<0.00001)$ and skin/nail changes, including alopecia, rashes, hand-foot syndrome and erythema (3.2 vs. $2.0 \%, \mathrm{P}=0.02)$. There was no statistical difference between other serious AEs (Table II). With respect to fatal AEs, there was no significant difference between fatal AEs in patients in the T- $(0.4 \%, 17 / 4,036)$ and $\mathrm{C}$-groups $(0.2 \%, 10 / 4,060, \mathrm{P}=0.19)$.

Serious AEs in a neoadjuvant setting. There was no statistical difference in the incidence of neutropenia, febrile neutropenia, 


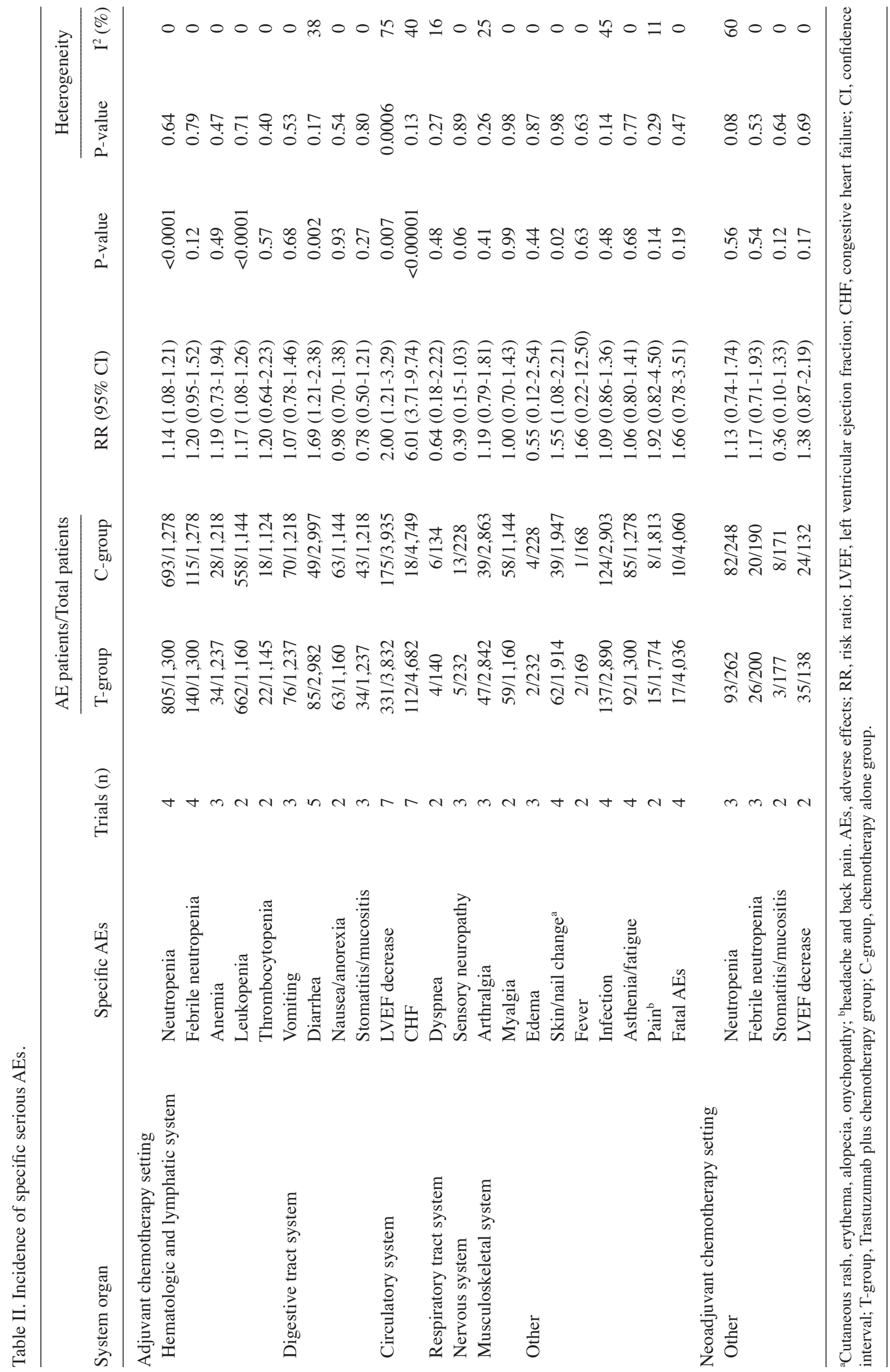


Table III. Relative risk of cardiac toxicity in patients with human epidermal growth factor receptor-2-positive breast cancer treated with adjuvant trastuzumab, stratified by timing.

\begin{tabular}{|c|c|c|c|c|c|c|c|}
\hline \multirow[b]{2}{*}{ Treatment } & \multirow[b]{2}{*}{ Trials (n) } & \multicolumn{2}{|c|}{ T-group (n) } & \multicolumn{2}{|c|}{ C-group (n) } & \multirow[b]{2}{*}{ RR $(95 \%$ CI $)$} & \multirow[b]{2}{*}{ P-value } \\
\hline & & Cardiac events & Total & Cardiac events & Total & & \\
\hline \multicolumn{8}{|l|}{ LVEF reduction } \\
\hline Concurrent & 5 & 240 & 1,890 & 155 & 1,948 & $1.54(1.27-1.86)$ & $<0.00001$ \\
\hline Sequential & 2 & 91 & 1,942 & 20 & 1,987 & $4.66(2.89-7.53)$ & $<0.00001$ \\
\hline Weekly & 3 & 45 & 771 & 41 & 856 & $1.16(0.77-1.76)$ & 0.47 \\
\hline Every 3 weeks & 3 & 92 & 2,019 & 20 & 2,061 & $4.62(2.88-7.41)$ & $<0.00001$ \\
\hline \multicolumn{8}{|l|}{$\mathrm{CHF}$} \\
\hline Concurrent & 5 & 75 & 2,740 & 15 & 2,762 & $4.79(2.80-8.17)$ & $<0.00001$ \\
\hline Sequential & 2 & 37 & 1,942 & 3 & 1,987 & $12.63(3.90-40.92)$ & $<0.0001$ \\
\hline Weekly & 3 & 53 & 1621 & 8 & 1670 & $3.34(0.55-20.44)$ & 0.19 \\
\hline Every 3 weeks & 3 & 38 & 2,019 & 3 & 2,061 & $11.2(3.76-33.35)$ & $<0.0001$ \\
\hline
\end{tabular}

LVEF, left ventricular ejection fraction; CHF, congestive heart failure; RR, risk ratio; CI, confidence interval; T-group, trastuzumab plus chemotherapy group; C-group, chemotherapy alone group.

Table IV. Publication bias accessed by Begg and Egger tests.

\begin{tabular}{lcc}
\hline Items & Begg's test & Egger's test \\
\hline Disease free survival & 1.000 & 0.904 \\
Overall survival & 0.711 & 0.682 \\
OR in adjuvant setting & 0.734 & 0.627 \\
pCR in neoadjuvant setting & 0.308 & 0.150 \\
LVEF reduction & 0.764 & 0.651 \\
Congestive heart failure & 0.548 & 0.562 \\
\hline
\end{tabular}

LVEF, left ventricular ejection fraction; OR, overall response; pCR, pathological complete response; LVEF, left ventricular ejection fraction.

stomatitis/microsites and LVEF reduction between the T- and C-groups. No mortalities were recorded as a result of treatment-related toxicities in the two groups (Table II).

Subgroup analysis of cardiac toxicity in an adjuvant setting. To further evaluate the cardiac toxicities of trastuzumab in an adjuvant setting, subgroup analysis was performed. Trastuzumab was assigned to concurrent chemotherapy in 5 trials $(\mathrm{n}=3,838) \quad(31,32,34,37,38)$, while 2 trials $(33,35)$ assigned sequential therapy $(\mathrm{n}=3,929)$. In the concurrent trials, the incidence of an LVEF decrease $(R R=1.54)$ and $\mathrm{CHF}(\mathrm{RR}=4.79)$ in the T-group was significantly higher than in the $\mathrm{C}$-group $(\mathrm{P}<0.00001)$. Similar results were observed in the sequential trials (LVEF, $\mathrm{RR}=4.66, \mathrm{P}<0.00001$; $\mathrm{CHF}$, $\mathrm{RR}=12.63, \mathrm{P}<0.0001)$. ITC demonstrated that the incidence of a reduction in LVEF in the concurrent trials was significantly lower than that in the sequential trials $(\mathrm{RR}=0.33, \mathrm{P}=0.00704)$. No significant difference was detected between the incidence of $\mathrm{CHF}$ in the concurrent and sequential trials $(\mathrm{RR}=0.379$, $\mathrm{P}=0.36255)$.
In trials where trastuzumab was administered weekly, the incidence of LVEF decrease $(R R=1.16)$ and $C H F(R R=3.34)$ was not significantly different between the $\mathrm{T}$ - and $\mathrm{C}$-groups $(\mathrm{P}=0.47$ and $\mathrm{P}=0.19$, respectively). However, the RR of LVEF decrease (4.62) and CHF (11.2) was significantly higher in the $\mathrm{C}$-group in comparison with the T-group when trastuzumab was administered every 3 weeks $(\mathrm{P}<0.00001, \mathrm{P}<0.0001$, respectively; Table III).

Publication bias. The sensitivity analysis revealed that there was no heterogeneity of recurrence and mortality once the joint study had been excluded from the analysis (32). This may have been caused by the slight design inconformity in individual trials. For example, patients with high-risk, node-negative BC were eligible to be included in the N9831, but not the B-31, trial, and the intention-to-treat analysis was not used in either of the 2 trials. No individual study affected the reduction in LVEF as the omission of any of the studies did not have an impact on the analysis. Funnel plots of DFS, OS, OR, LVEF reduction and CHF in an adjuvant setting, and PCR in a neoadjuvant setting, revealed no evident publication bias. The results were further confirmed by the Begg's and Egger's tests (Table IV).

\section{Discussion}

Trastuzumab serves a crucial function in the treatment of patients that are HER-2 positive. To evaluate the prognostic effects of trastuzumab, the AEs and administration timings of trastuzumab in a number of clinical trials in adjuvant and neoadjuvant settings for patients with HER-2 positive $B C$ were collected, and high quality RCTs published in recent years were included and analyzed in the present meta-analysis. In comparison with published meta-analyses which have reviewed trastuzumab treatment in patients with $\mathrm{BC}$ (Table V), a number of improvements were achieved in the current study. The present meta-analysis contained 13 RCTs with a total of 14,546 patients with HER-2 positive BC; to the best of our 


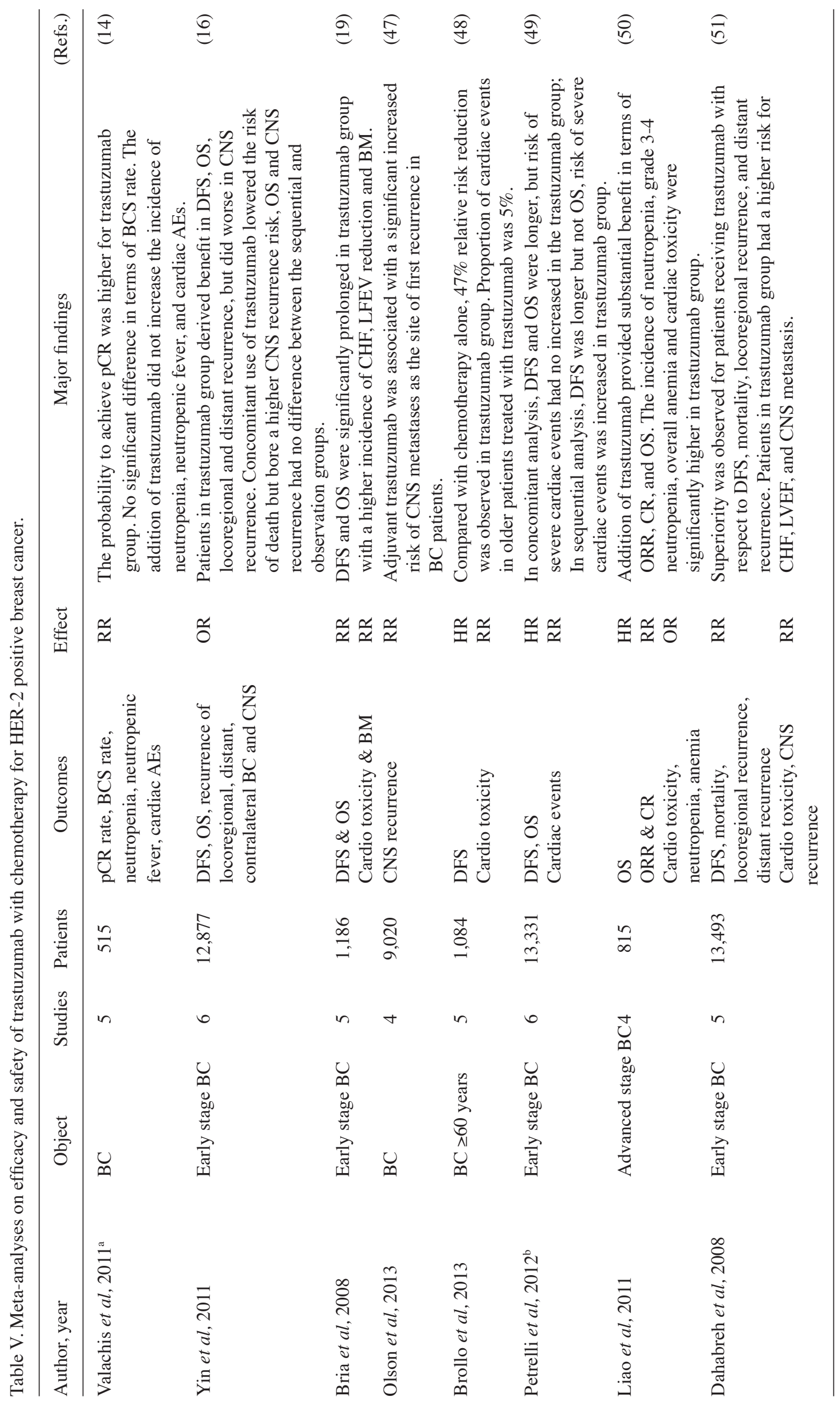


knowledge this is the largest number of samples included in a meta-analysis of this topic. In addition, an increased number of trials with longer follow-up periods were studied in the present meta-analysis in comparison with previous analyses.

The results from the present study demonstrated that the administration timings of trastuzumab can influence patient survival and cardiac toxicity. Aside from the most commonly studied cardiac toxicity, AEs of a number of systems and organs were analyzed in adjuvant and neoadjuvant settings in the present meta-analysis. DFS and OS may be regarded as the golden criteria to evaluate the long-term effects of trastuzumab, and the results from the current study demonstrated that the addition of trastuzumab in chemotherapy is able to increase DFS and OS in patients with HER-2 positive BC more effectively than in patients treated with chemotherapy alone, and similar results have been reported in previous meta-analyses (47-52). Notably, a number of subtle differences in the benefits of DFS and OS emerged when analyzing studies with extended follow-up periods. A joint analysis included in the present meta-analysis indicated that DFS and OS at 3 years follow-up were 87.1 and $94.3 \%$ in the T-group, and 75.4 and $91.7 \%$ in C-group, respectively (32); however, at 4 years, DFS and OS were 85.3 and $86.6 \%$ in the T-group, and 67.1 and $91.4 \%$ in the C-group, respectively. Compared with the $\mathrm{C}$-group, the benefit of DFS at 4-years in T-group still displayed statistical significance in the HERA trial, however, the OS benefit was no longer statistically significant by intention-to-treat analysis $(33,53)$. A previous meta-analysis demonstrated that the benefits of certain chemotherapies may improve after a 10- and 15-year follow up period (2). In summary, it was observed in the present study that long-term observation is required to determine the extent of the benefits of trastuzumab treatment in patients with HER-2 positive BC.

The subgroup analysis results from the present study determined that the optimum method of administering trastuzumab is to follow specific timings of administration in order to improve the DFS in patients with BC. In comparison with the C-group, the rate of OS improved in trials concurrently administering trastuzumab; however, this improvement was not observed in studies that administered trastuzumab sequentially. Notably, the present meta-analysis demonstrated that there is a significant improvement in DFS and OS when trastuzumab is administered weekly. By contrast, the administration of trastuzumab every 3 weeks improved DFS but not OS. In addition, ITC concluded that weekly trastuzumab administration improved the rate of DFS and OS compared with administration every 3 weeks. Survival analysis was not performed in the neoadjuvant setting due to the absence of mature data; therefore, it was not possible to arbitrarily conclude that the use of trastuzumab is beneficial for the survival outcomes of patients with HER-2 positive BC.

In an adjuvant setting, the OR was higher in the T-group in comparison with the C-group; however, the rates of SD and $\mathrm{PD}$ in the $\mathrm{C}$-group were higher compared with those in the T-group. This may be a result of the small sample size and the movement of patients in the C-groups of two studies $(37,38)$, who changed from receiving just chemotherapy to receiving chemotherapy plus trastuzumab prior to pre-specified endpoints. One of the two aforementioned studies (38) involved $56.4 \%$ (53/94) patients in C-group who crossed over to receive 
trastuzumab, which may have diluted some of the survival benefit conferred by trastuzumab. In addition, a number of patients in the T-group who responded to treatment were not followed-up after receiving the initial efficacy results.

With the exception of efficacy, AEs are important factors that should be taken into consideration when using trastuzumab. In an adjuvant setting, the present meta-analysis demonstrated that there are higher risks of grade 3-4 neutropenia, leukopenia, diarrhea, skin/nail change, LVEF reduction and $\mathrm{CHF}$ in T-groups in comparison with $\mathrm{C}$-groups; however, there was no difference in the presence of fatal AEs between the two groups. Fortunately, trastuzumab-associated AEs were manageable and reversible by discontinuing treatment (54-56). In the neoadjuvant setting, there was no statistical difference in the incidence of AEs between the two groups, and no mortalities resulting from trastuzumab-related toxicities were recorded. The present meta-analysis therefore indicates that AEs resulting from trastuzumab are manageable in adjuvant and neoadjuvant settings.

Cardiac toxicity is a common adverse reaction to trastuzumab and limits its use in patients with BC (20). Therefore, a detailed subgroup analysis of cardiac AEs resulting from trastuzumab administration was performed in an adjuvant setting. This analysis demonstrated that weekly administration of trastuzumab does not increase the incidence of LVEF reduction and $\mathrm{CHF}$, while the other three time-intervals (every 3 weeks, concurrent and sequential) may result in an increased risk of cardiac toxicity. ITC demonstrated that concurrent and weekly trastuzumab administration may result in fewer cardiac AEs in comparison with trastuzumab administered sequentially and every 3 weeks, and this was a similar result to that obtained by direct comparison. Although the results of ITC had not been seriously affected by the characteristics of patients, the quality, statistical methods and the intervention measures across trials, which are used as the evaluation standard of ITC analysis validity, differed between trials. In particular, the length of the follow-up period and the time frame of the RCTs may create bias within the results. Therefore, the results from the present study should be interpreted with caution.

There were a number of inconsistencies within the trials analyzed in this meta-analysis. The LVEF baseline recordings were obtained at different time points; for example, in one trial the baseline reading was recorded following completion of the primary treatment (which included surgery, radiotherapy and neoadjuvant or adjuvant chemotherapy, or both) (33), while in another trial the baseline reading was recorded prior to chemotherapy (36). The reported data regarding reduction in LVEF also had a number of inconsistencies; a decline of $>10 \%$ in LVEF was observed in 3 trials $(31,33,34),>15 \%$ in 3 trials $(32,35,38)$ and $>20 \%$ in one trial $(36)$. In addition, patients were considered ineligible if ventricular hypertrophy was detected on echocardiography in the NSABP B-31 trial, and poorly controlled hypertension and clinically significant pericardial effusion were exclusion criteria in the N9831 trial (57). In view of these inconsistencies, the results from the present study may have been different if the same criteria were applied to all RCTs analyzed.

A number limitations existed in the present study; although the Begg's and Egger's tests revealed no publication bias in the meta-analysis, the studies included in the analysis were conducted by different investigators from different institutions. Thus, potential publication bias remains a possibility. In addition, inconsistent methods of accessing the HER-2 status and different treatment schedules (weekly, every 3 weeks, concurrent and sequential) of patients may influence the accuracy of results. Furthermore, patients in the C-group changed to receive trastuzumab during the study; this may have reduced the survival advantage and treatment efficacy to an extent. Despite the limitations mentioned above, the prediction of the efficacy and safety of trastuzumab may be regarded as accurate and dependable in the present study.

In conclusion, the present meta-analysis supports the clinical practice of treating patients with HER-2 positive BC with a combination of trastuzumab and chemotherapy. AEs resulting from trastuzumab treatment were acceptable and manageable both in adjuvant and neoadjuvant settings. Furthermore, with regards to survival and cardiac toxicity, concurrent and weekly administration of trastuzumab has been demonstrated to be more effective than treatment with trastuzumab sequentially and every 3 weeks. Further research is required to confirm the findings of the present study and support the use of trastuzumab in clinical practice.

\section{Acknowledgements}

The present study was supported by the Project of the National Natural Science Foundation of China (grant no. 81572874)

\section{References}

1. Siegel R, Naishadham D and Jemal A: Cancer statistics, 2013. CA Cancer J Clin 63: 11-30, 2013.

2. Early Breast Cancer Trialists' Collaborative Group (EBCTCG): Effects of chemotherapy and hormonal therapy for early breast cancer on recurrence and 15-year survival: An overview of the randomised trials. Lancet 365: 1687-1717, 2005.

3. Mauri D, Pavlidis N and Ioannidis JP: Neoadjuvant versus adjuvant systemic treatment in breast cancer: A meta-analysis. J Natl Cancer Inst 3: 188-194, 2005.

4. Reese DM and Slamon DJ: HER-2/neu signal transduction in human breast and ovarian cancer. Stem Cells 15: 1-8, 1997.

5. Ross JS, Slodkowska EA, Symmans WF, Pusztai L, Ravdin PM and Hortobagyi GN: The HER-2 receptor and breast cancer: Ten years of targeted anti-HER-2 therapy and personalized medicine. Oncologist 14: 320-368, 2009.

6. Nabholtz JM and Gligorov J: Docetaxel/trastuzumab combination therapy for the treatment of breast cancer. Expert Opin Pharmacother 6: 1555-1564, 2005.

7. Slamon DJ, Godolphin W, Jones LA, Holt JA, Wong SG, Keith DE, Levin WJ, Stuart SG, Udove J and Ullrich A: Studies of the HER-2/neu proto-oncogene in human breast and ovarian cancer. Science 244: 707-712, 1989.

8. Slamon DJ, Clark GM, Wong SG, Levin WJ, Ullrich A and McGuire WL: Human breast cancer: Correlation of relapse and survival with amplification of the HER-2/neu oncogene. Science 235: 177-182, 1987.

9. Moasser MM: The oncogene HER2: Its signaling and transforming functions and its role in human cancer pathogenesis. Oncogene 26: 6469-6487, 2007.

10. Oostra DR and Macrae ER: Role of trastuzumab emtansine in the treatment of HER2-positive breast cancer. Breast Cancer (Dove Med Press) 6: 103-113, 2014.

11. Zhu ZL, Zhang J, Chen ML and Li K: Efficacy and safety of Trastuzumab added to standard treatments for HER2-positive metastatic breast cancer patients. Asian Pac J Cancer Prev 14: 7111-7116, 2013

12. Jelovac D and Wolff AC: The adjuvant treatment of HER2-positive breast cancer. Curr Treat Options Oncol 13: 230-239, 2012. 
13. Osoba D, Slamon DJ, Burchmore M and Murphy M: Effects on quality of life of combined trastuzumab and chemotherapy in women with metastatic breast cancer. J Clin Oncol 20 3106-3113, 2002.

14. Valachis A, Mauri D, Polyzos NP, Chlouverakis G, Mavroudis D and Georgoulias V: Trastuzumab combined to neoadjuvant chemotherapy in patients with HER2-positive breast cancer: A systematic review and meta-analysis. Breast 20: 485-490, 2011.

15. Rastogi P, Anderson SJ, Bear HD, Geyer CE, Kahlenberg MS, Robidoux A, Margolese RG, Hoehn JL, Vogel VG, Dakhil SR, et al: Preoperative chemotherapy: Updates of national surgical adjuvant breast and Bowel project protocols B-18 and B-27. J Clin Oncol 26: 778-785, 2008.

16. Yin W, Jiang Y, Shen Z, Shao Z and Lu J: Trastuzumab in the adjuvant treatment of HER2-positive early breast cancer patients: A meta-analysis of published randomized controlled trials. PLoS One 6: e21030, 2011.

17. Azim HA Jr, de Azambuja E, Paesmans $M$ and Piccart-Gebhart MJ: Sequential or concurrent administration of trastuzumab in early breast cancer? Too early to judge. J Clin Oncol 28: e353-e354, 2010.

18. Perez EA, Suman VJ, Davidson NE, Sledge GW, Kaufman PA, Hudis CA, Martino S, Gralow JR, Dakhil SR, Ingle JN, et al: Cardiac safety analysis of doxorubicin and cyclophosphamide followed by paclitaxel with or without trastuzumab in the north central cancer treatment group N9831 adjuvant breast cancer trial. J Clin Oncol 26: 1231-1238, 2008.

19. Bria E, Cuppone F, Fornier M, Nisticò C, Carlini P, Milella M, Sperduti I, Terzoli E, Cognetti F and Giannarelli D: Cardiotoxicity and incidence of brain metastases after adjuvant trastuzumab for early breast cancer: The dark side of the moon? A meta-analysis of the randomized trials. Breast Cancer Res Treat 109: 231-239, 2008.

20. Tan-Chiu E, Yothers G, Romond E, Geyer CE Jr, Ewer M, Keefe D, Shannon RP, Swain SM, Brown A, Fehrenbacher L, et al: Assessment of cardiac dysfunction in a randomized trial comparing doxorubicin and cyclophosphamide followed by paclitaxel, with or without trastuzumab as adjuvant therapy in node-positive, human epidermal growth factor receptor 2-overexpressing breast cancer: NSABP B-31. J Clin Oncol 23: 7811-7819, 2005 .

21. Tierney JF, Stewart LA, Ghersi D, Burdett S and Sydes MR Practical methods for incorporating summary time-to-event data into meta-analysis. Trials 8: 16, 2007.

22. Moher D, Liberati A, Tetzlaff J and Altman DG; PRISMA Group: Preferred reporting items for systematic reviews and meta-analyses: The PRISMA statement. BMJ 339: b2535, 2009 .

23. Holmes DR: Intraoperative radiotherapy in breast conserving surgery. J Surg Oncol 110: 68-74, 2014.

24. Higgins JP, Altman DG, Gøtzsche PC, Jüni P, Moher D, Oxman AD, Savovic J, Schulz KF, Weeks L and Sterne JA; Cochrane Bias Methods Group; Cochrane Statistical Methods Group: The Cochrane collaboration's tool for assessing risk of bias in randomised trials. BMJ 343: d5928, 2011.

25. Higgins JP, Thompson SG, Deeks JJ and Altman DG: Measuring inconsistency in meta-analyses. BMJ 327: 557-560, 2003.

26. Bucher HC, Guyatt GH, Griffith LE and Walter SD: The results of direct and indirect treatment comparisons in meta-analysis of randomized controlled trials. J Clin Epidemiol 50: 683-691, 1997.

27. Loke YK, Pradhan S, Yeong JK and Kwok CS: Comparative coronary risks of apixaban, rivaroxaban and dabigatran: A meta-analysis and adjusted indirect comparison. Br J Clin Pharmacol 78: 707-717, 2014.

28. Kang $\mathrm{N}$ and Sobieraj DM: Indirect treatment comparison of new oral anticoagulants for the treatment of acute venous thromboembolism. Thromb Res 133: 1145-1151, 2014

29. Egger M, Davey Smith G, Schneider M and Minder C: Bias in meta-analysis detected by a simple, graphical test. BMJ 315 629-634, 1997

30. Begg CB and Mazumdar M: Operating characteristics of a rank correlation test for publication bias. Biometrics 50 1088-1101, 1994.

31. Slamon D, Eiermann W, Robert N, Pienkowski T, Martin M, Press M, Mackey J, Glaspy J, Chan A, Pawlicki M, et al: Adjuvant trastuzumab in HER2-positive breast cancer. N Engl J Med 365: 1273-1283, 2011
32. Perez EA, Romond EH, Suman VJ, Jeong JH, Davidson NE, GeyerCE Jr, Martino S, Mamounas EP, Kaufman PA and Wolmark N: Four-year follow-up of trastuzumab plus adjuvant chemotherapy for operable human epidermal growth factor receptor 2-positive breast cancer: Joint analysis of data from NCCTG N9831 and NSABP B-31. J Clin Oncol 29: 3366-3373, 2011.

33. Gianni L, Dafni U, Gelber RD, Azambuja E, Muehlbauer S, Goldhirsch A, Untch M, Smith I, Baselga J, Jackisch C, et al: Treatment with trastuzumab for 1 year after adjuvant chemotherapy in patients with HER2-positive early breast cancer: A 4-year follow-up of a randomised controlled trial. Lancet Oncol 12: 236-244, 2011.

34. von Minckwitz G, du Bois A, Schmidt M, Maass N, Cufer T, de Jongh FE, Maartense E, Zielinski C, Kaufmann M, Bauer W, et al: Trastuzumab beyond progression in human epidermal growth factor receptor 2-positive advanced breast cancer: A german breast group 26/breast international group 03-05 study. J Clin Oncol 27: 1999-2006, 2009.

35. Spielmann M, Roché H, Delozier T, Canon JL, Romieu G, Bourgeois H, Extra JM, Serin D, Kerbrat P, Machiels JP, et al: Trastuzumab for patients with axillary-node-positive breast cancer: Results of the FNCLCC-PACS 04 trial. J Clin Oncol 27: 6129-6134, 2009.

36. Joensuu H,Bono P,Kataja V, Alanko T,KokkoR, Asola R, Utriainen T, Turpeenniemi-Hujanen T, Jyrkkiö S, Möykkynen $\mathrm{K}$, et al: Fluorouracil, epirubicin and cyclophosphamide with either docetaxel or vinorelbine, with or without trastuzumab, as adjuvant treatments of breast cancer: Final results of the FinHer Trial. J Clin Oncol 27: 5685-5692, 2009

37. Gasparini G, Gion M, Mariani L, Papaldo P, Crivellari D, Filippelli G, Morabito A, Silingardi V, Torino F, Spada A, et al: Randomized Phase II Trial of weekly paclitaxel alone versus trastuzumab plus weekly paclitaxel as first-line therapy of patients with Her-2 positive advanced breast cancer. Breast Cancer Res Treat 101: 355-365, 2007.

38. Marty M, Cognetti F, Maraninchi D, Snyder R, Mauriac L, Tubiana-Hulin M, Chan S, Grimes D, Antón A, Lluch A, et al: Randomized phase II trial of the efficacy and safety of trastuzumab combined with docetaxel in patients with human epidermal growth factor receptor 2-positive metastatic breast cancer administered as first-line treatment: The M77001 study group. J Clin Oncol 23: 4265-4274, 2005.

39. Slamon DJ, Leyland-Jones B, Shak S, Fuchs H, Paton V, Bajamonde A, Fleming T, Eiermann W, Wolter J, Pegram M, et al: Use of chemotherapy plus a monoclonal antibody against HER2 for metastatic breast cancer that overexpresses HER2. N Engl J Med 344: 783-792, 2001.

40. Steger GG, Greil R, Lang A, Rudas M, Fitzal F, Mlineritsch B, Hartmann BL, Bartsch R, Melbinger E, Hubalek M, et al: Epirubicin and docetaxel with or without capecitabine as neoadjuvant treatment for early breast cancer: Final results of a randomized phase III study (ABCSG-24). Ann Oncol 25: 366-371, 2014

41. Pierga JY, Delaloge S, Espié M, Brain E, Sigal-Zafrani B, Mathieu MC, Bertheau P, Guinebretière JM, Spielmann M, Savignoni A and Marty M: A multicenter randomized phase II study of sequential epirubicin/cyclophosphamide followed by docetaxel with or without celecoxib or trastuzumab according to HER 2 status, as primary chemotherapy for localized invasive breast cancer patients. Breast Cancer Res Treat 122: 429-437, 2010.

42. Gianni L, Eiermann W, Semiglazov V, Manikhas A, Lluch A, Tjulandin S, Zambetti M, Vazquez F, Byakhow M, Lichinitser M, et al: Neoadjuvant chemotherapy with trastuzumab followed by adjuvant trastuzumab versus neoadjuvant chemotherapy alone, in patients with HER2-positive locally advanced breast cancer (the NOAH trial): A randomised controlled superiority trial with a parallel HER2-negative cohort. Lancet 375: 377-384, 2010.

43. Buzdar AU, Valero V, Ibrahim NK, Francis D, Broglio KR, Theriault RL, Pusztai L, Green MC, Singletary SE, Hunt KK, et al: Neoadjuvant therapy with paclitaxel followed by 5-fluorouracil, epirubicin and cyclophosphamide chemotherapy and concurrent trastuzumab in human epidermal growth factor receptor 2-positive operable breast cancer: An update of the initial randomized study population and data of additional patients treated with the same regimen. Clin Cancer Res 13: 228-233, 2007.

44. Buzdar AU, Ibrahim NK, Francis D, Booser DJ, Thomas ES, Theriault RL, Pusztai L, Green MC, Arun BK, Giordano SH, et al: Significantly higher pathologic complete remission rate after neoadjuvant therapy with trastuzumab, paclitaxel and epirubicin chemotherapy: Results of a randomized trial in human epidermal growth factor receptor 2-positive operable breast cancer. J Clin Oncol 23: 3676-3685, 2005 
45. World Health Organisation: WHO handbook for reporting results of cancer treatment. WHO offset publication no 48 . World Health Organisation, Geneva, Switzerland, 1979.

46. Khokher S, Qureshi MU and Chaudhry NA: Comparison of WHO and RECIST criteria for evaluation of clinical response to chemotherapy in patients with advanced breast cancer. Asian Pac J Cancer Prev 13: 3213-3218, 2012.

47. Olson EM, Abdel-Rasoul M, Maly J, Wu CS, Lin NU and Shapiro CL: Incidence and risk of central nervous system metastases as site of first recurrence in patients with HER2-positive breast cancer treated with adjuvant trastuzumab. Ann Oncol 24: 1526-1533, 2013.

48. Brollo J, Curigliano G, Disalvatore D, Marrone BF, Criscitiello C, Bagnardi V, Kneubil MC, Fumagalli L, Locatelli M, Manunta S and Goldhirsch A: Adjuvant trastuzumab in elderly with HER-2 positive breast cancer: A systematic review of randomized controlled trials. Cancer Treat Rev 39: 44-50, 2013.

49. Petrelli F and Barni S: Meta-analysis of concomitant compared to sequential adjuvant trastuzumab in breast cancer: The sooner the better. Med Oncol 29: 503-510, 2012.

50. Liao C, Yin F, Huang P, Cao Y and Gao F: A meta-analysis of randomized controlled trials comparing chemotherapy plus trastuzumab with chemotherapy alone in HER-2-positive advanced breast cancer. Breast J 17: 109-111, 2011.

51. Dahabreh IJ, Linardou H, Siannis F, Fountzilas G and Murray S: Trastuzumab in the adjuvant treatment of early-stage breast cancer: A systematic review and meta-analysis of randomized controlled trials. Oncologist 13: 620-630, 2008.
52. Moja L, Tagliabue L, Balduzzi S, Parmelli E, Pistotti V, Guarneri $\mathrm{V}$ and D'Amico R: Trastuzumab containing regimens for early breast cancer. Cochrane Database Syst Rev 4: CD006243, 2012.

53. Piccart-Gebhart MJ, Procter M, Leyland-Jones B, Goldhirsch A, Untch M, Smith I, Gianni L, Baselga J, Bell R, Jackisch C, et al: Trastuzumab after adjuvant chemotherapy in HER2-positive breast cancer. N Engl J Med 353: 1659-1672, 2005.

54. Russell SD, Blackwell KL, Lawrence J, Pippen JE Jr, Roe MT, Wood F, Paton V, Holmgren E and Mahaffey KW: Independent adjudication of symptomatic heart failure with the use of doxorubicin and cyclophosphamide followed by trastuzumab adjuvant therapy: A combined review of cardiac data from the national surgical adjuvant breast and Bowel project B-31 and the north central cancer treatment group N9831 clinical trials. J Clin Oncol 28: 3416-3421, 2010.

55. Procter M, Suter TM, de Azambuja E, Dafni U, van Dooren V, Muehlbauer S, Climent MA, Rechberger E, Liu WT, Toi M, et al: Longer-term assessment of trastuzumab-related cardiac adverse events in the Herceptin adjuvant (HERA) trial. J Clin Oncol 28: 3422-3428, 2010.

56. de Azambuja E, Bedard PL, Suter T and Piccart-Gebhart M: Cardiac toxicity with anti-HER-2 therapies: What have we learned so far? Target Oncol 4: 77-88, 2009.

57. Romond EH, Perez EA, Bryant J, Suman VJ, Geyer CE Jr, Davidson NE, Tan-Chiu E, Martino S, Paik S, Kaufman PA, et al: Trastuzumab plus adjuvant chemotherapy for operable HER2-positive breast cancer. N Engl J Med 353: 1673-1684, 2005. 